\title{
A STOCHASTIC OVERLAPPING GENERATION MODEL WITH A CONTINUUM OF AGENTS
}

\author{
EMMANUELLE AUGERAUD-VÉRON and DELPHINE DAVID \\ Laboratoire de Mathématiques, Université de La Rochelle \\ Avenue Michel Crépeau, 17042 La Rochelle Cedex, France \\ E-mail: eaugeraud@univ-lr.fr,delphine.david@univ-lr.fr
}

\begin{abstract}
We consider a stochastic overlapping generations model for a continuum of individuals with finite lives in presence of a financial market. In this paper, an agent's heterogeneity is given by the dates of birth of the household members, in contrast to standard models, in which each agent has his own aversion coefficient on his utility function. By means of martingale arguments, we compute the agent's optimal consumption and portfolio. A characterization of interest rate trajectories is given by mixed-type functional differential equations and the stability of these trajectories is studied.
\end{abstract}

1. Introduction. We consider an OverLapping Generations (OLG) framework for a continuum of individuals in the presence of a financial market. In non-OLG models, as in [13] for example, an agent's heterogeneity appears through the different relative risk aversion coefficients, the individuals commonly live on a finite time interval and the economy contains a finite number of agents. On the contrary, in OLG models, an agent's heterogeneity appears through the demographic structure, the individuals are characterized by their birth date and a continuum of agents is considered. Furthermore, the economy lives on an infinite time horizon and the number of agents is unknown.

The main goal of this paper is the study of interest-rates and prices behaviors on different markets. Our approach is a stochastic extension of the general equilibrium for deterministic OLG models developed in particular by Polemarchakis and Demichelis [19] and by d'Albis and Augeraud-Véron [6]. In these cases, the intertemporal equilibrium is the solution of a mixed type functional differential equation (MFDE). We show that

2000 Mathematics Subject Classification: 91B70, 91B62, 91B42.

JEL Classification: G11, E44, D91.

Key words and phrases: stochastic overlapping generations, mixed-type functional differential equations.

The paper is in final form and no version of it will be published elsewhere. 
the stochastic extension preserves this MFDE. Some results on mixed type and retarded functional differential equations are presented in the appendix section.

In Section 2, we compute the optimal consumption/investment strategy of a household. This type of problem has been widely studied in the last few decades. In two papers, Merton [17, 18] introduced a model with constant coefficients and solved the associated Hamilton-Jacobi-Bellman equation. Bismut [3] obtained formulas for optimal consumption using his stochastic duality theory (Bismut [2]). We also refer to Lehoczky, Sethi and Shreve [15], Karatzas, Lehoczky and Shreve [10] and Cox and Huang [5] for similar results.

In Section 3, we present our market equations and give conditions for existence of steady states. These conditions recover in particular the ones of d'Albis and AugeraudVéron for the deterministic case. The multiple steady states we obtain are possible under conditions relying on endowment distribution similar to those proposed by Kim [14]. In the standard stochastic equilibrium model, as in [9], [11] and [12], the endowment of agents is an exogeneous process and individuals wish to hedge the variability in their endowment processes by trading with one another. This justifies the introduction of a financial market. Applying this setting to our stochastic overlapping generations model leads to very complex equations. Consequently, deterministic wages are considered instead of stochastic endowment. For this reason, this model can't be seen as an equilibrium model.

Section 4 is dedicated to the local dynamics of the interest rates around the steadystates we have obtained. This stability analysis gives us the behaviors of the trajectories and uses the theory of MFDE, in particular the recent results of Mallet-Paret and Verduyn-Lunel [16].

2. The model. We consider a standard complete financial market with two assets. One of them is a non risky asset with price per unit $B(t)$ governed by the equation:

$$
d B(t)=r(t) B(t) d t, \quad B(0)=1,
$$

where $r(t)$ is the interest rate. The second asset is a risky one with price process $(S(t)$, $t \geq 0)$ defined by the following stochastic differential equation:

$$
d S(t)=\mu(t) S(t) d t+\sigma(t) S(t) d W(t), \quad S(0)=x,
$$

where $W$ is a standard, one-dimensional Brownian motion on $\mathbb{R}$, defined on a probability space $(\Omega, \mathcal{F}, P)$ equipped with the filtration $\left(\mathcal{F}_{t}\right)$ generated by the Brownian motion $W$ and augmented. Throughout the paper, we assume that $r(\cdot), \mu(\cdot)$ and $\sigma(\cdot)$ are deterministic functions. The economy is a pure exchange one with a single perishable good and we assume that the quantity produced in the economy is normalized to 1 .

Individual setting. We consider an agent born at time $s \in \mathbb{R}^{+}$and living for a time interval of a unit length. During his lifetime, he receives a wage $w(s, t)$ defined by

$$
w(s, t)=\left\{\begin{array}{l}
w \quad \text { if } t \in[s+\alpha, s+\beta], \\
0 \quad \text { otherwise }
\end{array}\right.
$$


with $0 \leq \alpha<\beta \leq 1$ and $w$ a constant. At each time $t \in[s, s+1]$, he chooses the proportion of wealth $\pi(s, t)$ he invests in the risky asset and his consumption $c(s, t)$.

Definition 2.1. The consumption process of an individual born at time $s$ is an $\left\{\mathcal{F}_{t}\right\}$ progressively mesurable, nonnegative process $c(s,$.$) , satisfying$

$$
\int_{s}^{s+1} c(s, t) d t<\infty, \quad \forall s \in \mathbb{R}^{+},
$$

and

$$
\int_{t-1}^{t} c(s, t) d s<\infty, \quad \forall t \in \mathbb{R}^{+}
$$

almost surely.

Denoting by $X(s, t)$ his wealth at time $t$, his budget constraint reads:

$$
\begin{aligned}
d X(s, t)= & {[(r(t)+\pi(s, t)(\mu(t)-r(t)) X(s, t)-c(s, t)+w(s, t)] d t} \\
& +\sigma(t) \pi(s, t) X(s, t) d W(t), \\
X(s, s)= & 0 .
\end{aligned}
$$

Utility functions. We denote by $\mathbb{R}_{+}^{*}$ the set $\{x \in \mathbb{R} ; x>0\}$. Denote by $U(s)$ the intertemporal utility of an individual born at time $s \geq 0$ and given by:

$$
U(s)=\mathbb{E}\left[\int_{s}^{s+1} e^{-\rho(t-s)} u(c(s, t)) d t \mid \mathcal{F}_{s}\right]
$$

where $u(\cdot)$ is a utility function and $\rho \geq 0$ is the discount rate.

Definition 2.2. A utility function is a concave, nondecreasing, upper semicontinuous function $u: \mathbb{R}_{+}^{*} \rightarrow \mathbb{R}$ satisfying:

- the half-line $\operatorname{dom}(u) \triangleq\left\{x \in \mathbb{R}_{+}^{*} ; u(x)>-\infty\right\}$ is a nonempty subset of $[0, \infty)$;

- $u^{\prime}$ is continuous, positive, and strictly decreasing on the interior of $\operatorname{dom}(u)$, and

$$
u^{\prime}(\infty) \triangleq \lim _{x \rightarrow \infty} u^{\prime}(x)=0
$$

- the Inada condition holds:

$$
u^{\prime}(0) \triangleq \lim _{x \rightarrow 0} u^{\prime}(x)=\infty .
$$

The strictly decreasing, continuous function $u^{\prime}:(0, \infty) \stackrel{\text { onto }}{\longrightarrow}\left(0, u^{\prime}(0)\right)$ has a strictly decreasing, continuous inverse $I:\left(0, u^{\prime}(0)\right) \stackrel{\text { onto }}{\rightarrow}(0, \infty)$. We set $I(y)=0$ for $U^{\prime}(0) \leq y \leq \infty$.

Definition 2.3. Let $u$ be a utility function. The convex dual of $u$ defined on $\mathbb{R}_{+}^{*}$ is the function

$$
\tilde{u}(y) \triangleq \sup _{x \geq 0}\{u(x)-x y\} .
$$

The convex dual $\tilde{u}$ is in fact the Legendre-Fenchel transform of $u$ except for the minus sign. By Lemma 4.3, Chapter 3 of [13] we have:

$$
\tilde{u}(y)=u(I(y))-y I(y) \text { if } y>0 .
$$


The optimization problem. The individual program is to maximize (2.2) subject to (2.1). In the sequel, we will consider the particular case of $u(c(s, t))=c(s, t)^{1-1 / \gamma} /(1-1 / \gamma)$ where $\gamma>0$ stands for the elasticity of intertemporal substitution. Using the martingale approach, it is well-known that we can transform our constraint into the following one:

$$
\mathbb{E}\left[\int_{s}^{s+1} \frac{H(u)}{H(s)} c(s, u) d u \mid \mathcal{F}_{s}\right]=\mathbb{E}\left[\int_{s}^{s+1} \frac{H(u)}{H(s)} w(s, u) d u \mid \mathcal{F}_{s}\right]
$$

where $H(t)=\exp \left\{-\int_{0}^{t} r(\tau)+\frac{1}{2} \theta(\tau)^{2} d \tau-\int_{0}^{t} \theta(\tau) d W(\tau)\right\}$ and the Sharpe ratio is $\theta(t)=$ $(\mu(t)-r(t)) / \sigma(t)$, see Karatzas and Shreve [13], Chapter 3, for more details.

Proposition 2.4. The optimal consumption and portfolio process pair $\left(c^{*}, \pi^{*}\right)$ is given by

$$
\begin{aligned}
& c^{*}(s, t)=e^{\rho(t-s)}\left(\frac{H(t)}{H(s)}\right)^{-\gamma} \frac{\int_{s}^{s+1} \frac{B(s)}{B(u)} w(s, u) d u}{\int_{s}^{s+1}\left(\frac{B(s)}{B(u)}\right)^{1-\gamma} e^{-\frac{\gamma}{2}(1-\gamma) \int_{s}^{u} \theta(\tau)^{2} d \tau+\gamma \rho(u-s)} d u}, \\
& \pi^{*}(s, t)=\frac{\Psi(s, t) H(s)}{\sigma(t) X(s, t) H(t)}+\frac{\theta(t)}{\sigma(t)},
\end{aligned}
$$

where $\Psi(s, t)$ is the integrand in the stochastic integral representation $M(s, t)=$ $\int_{s}^{t} \Psi(s, u) d W(u)$ of the martingale

$$
M(s, t) \triangleq \mathbb{E}\left[\int_{s}^{s+1} \frac{H(u)}{H(s)}(c(s, u)-w(s, u)) d u \mid \mathcal{F}_{t}\right] .
$$

Proof. The proof can be found in [13], Chapter 3, Section 3.6 and 3.7.

3. Prices and interest rates behaviors. In this section, we give a characterization of the behaviors of prices and interest rate considering equalities on different markets. These equalities can be seen as simplifications of equilibrium equations in the sense that we represent the demand/supply equalities in expectation.

Market equations. First of all, we introduce the equalities on different markets. This definition is adapted from Definition 5.1, Chapter 4 of [13].

Definition 3.1. A vector $\left(r^{*}(t), \theta^{*}(t)\right)$ is a solution of the market equations if:

1. The pair $\left(c^{*}, \pi^{*}\right)$ is optimal for each individual.

2. The markets clear:

$$
\begin{aligned}
& \mathbb{E}\left[\int_{t-1}^{t} c^{*}(s, t) d s\right]=\int_{t-1}^{t} w(s, t) d s, \\
& \mathbb{E}\left[\int_{t-1}^{t} \pi^{*}(s, t) X(s, t) d s\right]=0, \\
& \int_{t-1}^{t} w(s, t) d s=1 .
\end{aligned}
$$

The interpretation for the market equations is the following: At all times the aggregate wage is consumed as it enters the economy and the asset is in zero net supply. The first condition is due to the perishable good, the second one reflects the fact that for every buyer in the financial market, there must be a seller. 
This definition can be seen as a restriction for our model. However, this permits us to obtain explicit solutions for prices, otherwise this is not the case. These equations give the result below.

THEOREM 3.2. The vector $\left(r^{*}(t), \theta^{*}(t)\right)$ is a solution of market equations if and only if, for all $t \in \mathbb{R}^{+}$:

$$
\int_{t-1}^{t} e^{-\gamma \rho(t-s)}\left(\frac{B(s)}{B(t)}\right)^{-\gamma} \frac{e^{\frac{\gamma}{2}(1+\gamma) \int_{s}^{t} \theta^{*}(\tau)^{2} d \tau} \int_{s}^{s+1} \frac{B(s)}{B(u)} w(s, u) d u}{\int_{s}^{s+1}\left(\frac{B(s)}{B(u)}\right)^{1-\gamma} e^{-\frac{\gamma}{2}(1-\gamma) \int_{s}^{u} \theta^{*}(\tau)^{2} d \tau-\gamma \rho(u-s)} d u} d s=1
$$

and

$$
\begin{aligned}
& \frac{\theta(t)}{\sigma(t)}\left[\int_{t-1}^{t} \int_{t}^{s+1} \frac{B(t)}{B(u)} w(s, u) d u d s-(1-\gamma) \int_{t-1}^{t}\left(\frac{B(s)}{B(t)}\right)^{-\gamma} e^{\frac{\gamma}{2}(1+\gamma) \int_{s}^{t} \theta^{*}(\tau)^{2} d \tau}\right. \\
& \left.\times \int_{s}^{s+1} \frac{B(s)}{B(u)} w(s, u) d u \frac{\int_{t}^{s+1}\left(\frac{B(t)}{B(u)}\right)^{1-\gamma} e^{-\frac{\gamma}{2}(1-\gamma) \int_{t}^{u} \theta^{*}(\tau)^{2} d \tau-\gamma \rho(u-s)} d u}{\int_{s}^{s+1}\left(\frac{B(s)}{B(u)}\right)^{1-\gamma} e^{-\frac{\gamma}{2}(1-\gamma) \int_{s}^{u} \theta^{*}(\tau)^{2} d \tau-\gamma \rho(u-s)} d u} d s\right]=0
\end{aligned}
$$

where $B(t)=\exp \left\{\int_{0}^{t} r^{*}(s) d s\right\}$. Moreover, equation (3.3) gives $w=1 /(\beta-\alpha)$.

Proof. The first part of this result is obtained by computing the expectations and using the martingale property of $\exp \left\{\int_{s}^{t} \theta^{*}(\tau) d W(\tau)-\frac{1}{2} \int_{s}^{t} \theta^{*}(\tau)^{2} d \tau\right\}$. For the second part we use the definition of $w(s, t)$ which is $w(s, t)=w \mathbf{1}_{[s+\alpha, s+\beta]}(t)$ with $0 \leq \alpha<\beta \leq 0$.

Steady states. The goal is now to determine the conditions of existence and multiplicity of steady states for our market equations.

Definition 3.3. A time-independent vector $(r(t), \theta(t))=\left(r^{*}, \theta^{*}\right)$ satisfying equations (3.4) and (3.5) is called a steady state.

The first result is the following:

LEMMA 3.4. For all steady state vectors $\left(r^{*}, \theta^{*}\right)$ we have

$$
\frac{\int_{0}^{1} e^{\gamma r^{*} \tau+\rho \tau+\frac{\gamma}{2}(1+\gamma) \theta^{* 2} \tau} d \tau \int_{0}^{1} e^{-r^{*} \tau-\theta^{*} \kappa \tau} d \tau}{\int_{0}^{1} e^{-(1-\gamma) r^{*} \tau-\frac{\gamma}{2}(1-\gamma) \theta^{* 2} \tau+\rho \tau} d \tau}=\int_{0}^{1} e^{\nu \tau} d \tau
$$

and at least one of these equations are satisfied:

$$
\begin{gathered}
\bullet \theta_{\alpha}^{*}=0 \\
\int_{u}^{\beta} e^{r^{*} u} \int_{u}^{\beta} e^{-r^{*} \tau} d \tau d s+\int_{0}^{\alpha} e^{r^{*} u} \int_{\alpha}^{\beta} e^{-r^{*} \tau} d \tau d s-(1-\gamma) \int_{0}^{1}\left(e^{r^{*} u+\gamma \theta^{* 2} u} \int_{\alpha}^{\beta} e^{-r^{*} \tau} d \tau\right. \\
\left.\times \frac{\int_{u}^{1} e^{-(1-\gamma) r^{*} \tau-\frac{\gamma}{2}(1-\gamma) \theta^{* 2} \tau-\gamma \rho \tau} d \tau}{\int_{0}^{1} e^{-(1-\gamma) r^{*} \tau-\frac{\gamma}{2}(1-\gamma) \theta^{* 2} \tau-\gamma \rho \tau} d \tau}\right) d s=0 .
\end{gathered}
$$

REMARKS 3.5. 1. We obtain steady states which are the same as the solutions which can be found in non-OLG equilibrium models.

2. $\theta^{*}=0$ is the unique solution if $\gamma \geq 1$. 
In the sequel we focus on the case $\theta^{*}=0$ and study solutions to equation (3.6). To simplify the notation, we drop the star indices and define

$$
\Phi(r ; \alpha, \beta, \gamma, \rho)=\frac{\int_{0}^{1} e^{\gamma r s+\gamma \rho s} d s \int_{\alpha}^{\beta} e^{-r s} d s}{(\beta-\alpha) \int_{0}^{1} e^{-(1-\gamma) r s+\gamma \rho s} d s}
$$

which is obtained by replacing $\theta$ by 0 in equation (3.6). When there is no ambiguity for the parameter values, we denote $\Phi(r ; \alpha, \beta, \gamma \rho)=\Phi(r)$.

Property 3.6. A steady state is an $r$ that satisfies $\Phi(r)=1$ if $r \neq 0$ and $\Phi^{\prime}(r)=0$ if $r=0$.

Proof. For $r \neq 0$, the property is an immediate implication of lemma 3.4. For $r=0$, we just observe that $\Phi(0)=1$ and use l'Hôpital's Rule.

To obtain $r=0$ as a solution, we have to make some restrictions on the parameters:

Proposition 3.7. $r=0$ is a steady state if and only if

$$
\begin{cases}\alpha+\beta=2\left(\frac{e^{\gamma \rho}(\gamma \rho-1)+1}{\gamma \rho\left(e^{\gamma \rho}-1\right)}\right) & \text { if } \rho \neq 0 \\ \alpha+\beta=1 & \text { if } \rho=0 .\end{cases}
$$

Proof. The first derivative of function $\Phi$ yields:

$$
\Phi^{\prime}(r)=\Phi(r)\left[\gamma \frac{\int_{0}^{1} s e^{\gamma r s+\gamma \rho s} d s}{\int_{0}^{1} e^{\gamma \rho s} d s}-\frac{\int_{\alpha}^{\beta} s e^{-r s} d s}{\int_{\alpha}^{\beta} e^{-r s} d s}+(1-\gamma) \frac{\int_{0}^{1} s e^{-(1-\gamma) r s+\gamma \rho s} d s}{\int_{0}^{1} e^{-(1-\gamma) r s+\gamma \rho s} d s}\right] .
$$

Replacing $r=0$ in $\Phi^{\prime}(r)$, we conclude directly.

The two following propositions present the existence and multiplicity of the steady states for equation (3.6).

Proposition 3.8. There exists $(\alpha, \beta, \rho, \gamma)$ such that there is no steady state.

Proof. We show that there is no $r$ such that $\Phi(r)=1$ if $\alpha=0$ and $\beta \in(0,1-\gamma)$ with $\rho=0$. Define $\Psi(\beta)=\Phi(r ; 0, \beta, \gamma, 0)$. Then

$$
\Psi(\beta)=\frac{\int_{0}^{1} e^{\gamma r s} d s \int_{0}^{\beta} e^{-r s} d s}{\beta \int_{0}^{1} e^{-(1-\gamma) r s} d s}
$$

and

$$
\Psi^{\prime}(\beta)=\left[\frac{r}{e^{r \beta}-1}-\frac{1}{\beta}\right] \Psi(\beta) .
$$

Consequently, if $r>0$ we verify that $\Psi^{\prime}(\beta)<0$ and $\lim _{\beta \rightarrow 1-\gamma} \Psi(\beta)>1$. Conversely, if $r<0, \Psi^{\prime}(\beta)>0$ and $\lim _{\beta \rightarrow 0} \Psi(\beta)>1$. This concludes the proof. Notice that it can also be shown that there is no solution $r$ such that $\Phi(r)=1$ if $\beta=1$ and $\alpha \in[\gamma, 1)$.

Proposition 3.9. There exists $(\alpha, \beta, \rho, \gamma)$ such that there are multiple steady states.

Proof. Suppose $\beta=1-\alpha$ and $\rho=0$. With Proposition 3.7, $r=0$ is a steady state. We show that there are at least two other solutions such that $\Phi(r)=1$ if $\gamma \in(\alpha, 2 \alpha(1-\alpha))$. We have

$$
\lim _{r \rightarrow-\infty} \frac{\Phi^{\prime}(r)}{\Phi(r)}=\gamma-\alpha \text { and } \lim _{r \rightarrow+\infty} \frac{\Phi^{\prime}(r)}{\Phi(r)}=\alpha-\gamma .
$$


Consequently, for $\gamma>\alpha$

$$
\lim _{r \rightarrow \pm \infty} \Phi(r)=+\infty
$$

Moreover, $\Phi^{\prime}(0)=0$, and since

$$
\Phi^{\prime \prime}(0)=\frac{1}{6}(\gamma-2 \alpha(1-\alpha)),
$$

then $\Phi^{\prime \prime}(0)<0$, for $\gamma<2 \alpha(1-\alpha)$.

4. Stability analysis. In this section we are interested in the local dynamics around the steady state $r^{*}=0$. Let us define $x(t)$ such that $r(t)=r^{*}+\varepsilon x(t)$. We take $\rho=0$, then from Proposition 3.7, $\beta=1-\alpha$.

LEMMA 4.1. The function $x(\cdot)$ satisfies the following MFDE:

$$
\begin{aligned}
\int_{t-1}^{t}\left[(1-2 \alpha)\left(\gamma \int_{s}^{t} x(u) d u+(1-\gamma) \int_{s}^{s+1} \int_{s}^{u} x(\tau) d \tau d u\right)\right. & \\
& \left.-\int_{s+\alpha}^{s+1-\alpha} \int_{s}^{u} x(\tau) d \tau d u\right] d s=0 .
\end{aligned}
$$

Proof. The result is obtained by replacing $r(t)$ by $r^{*}+\varepsilon x(t)$ in equation (4.1) and by taking a Taylor expansion in the neighbourhood of $\varepsilon=0$.

In the sequel, to simplify, we take $\alpha=0$. In this case, the new equation is:

$$
\int_{t-1}^{t}\left[\int_{s}^{t} x(u) d u-\int_{s}^{s+1} \int_{s}^{u} x(\tau) d \tau d u\right] d s=0 .
$$

We now apply the results presented in Section 2 for our equation (4.2).

Stability of equation (4.1). The characteristic function associated to equation (4.2) is:

$$
\Delta(\lambda)=\frac{-\lambda^{2}+e^{\lambda}-2+e^{-\lambda}}{\lambda^{3}} .
$$

First, note that the roots of this function are symmetric with respect to both coordinate axes. Defining $\Lambda_{U}=\{\lambda \in \mathbb{C} ; \Delta(\lambda)=0$ and $\operatorname{Re}(\lambda)>0\}$ and $\Lambda_{S}=\{\lambda \in \mathbb{C} ; \Delta(\lambda)=0$ and $\operatorname{Re}(\lambda)<0\}$, the following lemma holds:

Lemma 4.2. If $\lambda \in \Lambda_{U},-\lambda \in \Lambda_{S}$.

We use the factorization result of Mallet-Paret and Verduyn Lunel [16] and remark that Proposition 2.1 can be applied. So we obtain this fundamental theorem:

THEOREM 4.3. Let $x(t)$ with $t \in[-1,0]$. There exists a unique, bounded and continuous trajectory $x(t), t \in \mathbb{R}^{+}$, such that

$$
\frac{d^{2} x(t)}{d t^{2}}=x(t+1)-2 x(t)+x(t-1) .
$$

Proof. This result is obtained by differentiating three times the first equation and using the exponential dichotomies result of Mallet-Paret and Verduyn-Lunel [16]. 
Comments. According to our stability analysis, the mixed-type functional differential equation theory tells us that there are oscillations around the steady states for the trajectory $x(t)$. Our steady state was for $\theta^{*}=0$, so we have $r^{*}=\mu^{*}$. Denoting $\mu(t)$ as $\mu^{*}+\epsilon y(t)$, the dynamics of $y(t)$ are associated to the ones of $x(t)$ and we obtain the same oscillations for $y(t)$ as for $x(t)$. These oscillations decrease in magnitude and eventually disappear.

5. Conclusion. We considered a stochastic overlapping generations model in which the shock in the economy comes only from the risky asset on the financial market. In this approach, we obtained steady states which were very similar to the equilibrium solutions for non-OLG models. We added asymptotic results for an exchange economy without any terminal time and for heterogeneous agents. In this paper, we focused on the steady state $r^{*}=0$ and $\theta^{*}=0$. The dynamics of $r(\cdot)$ were given by a functional differential equation of mixed type, due to the anticipation behavior in the individual program and to the demographic modelling. The study of these dynamics provided replacement echoes, which are usual in models with delays as in Boucekkine, Germain and Licandro [4].

6. Appendix. In this appendix section, we present the theory of MFDE we use in Section 5. Results for advanced and retarded equations come from Mallet-Paret and Verduyn Lunel [16].

Let us consider the following linear functional differential equation of mixed type:

$$
\dot{x}(t)=\int_{-1}^{1} x(t+\theta) d \eta(\theta)
$$

where $d \eta(\theta)$ is a finite Lebesgue-Stieltjes measure on $[-1,1]$.

Replacing $x(t)=e^{\lambda t}, \quad \lambda \in \mathbb{C}$ in (6.1), we can define the characteristic function as:

$$
\Delta(\lambda)=\lambda-\int_{-1}^{1} e^{\lambda \theta} d \eta(\theta) .
$$

In their paper, Mallet-Paret and Verduyn Lunel obtain a factorization of the characteristic function (6.2):

$$
\Psi(\lambda) \Delta(\lambda)=\Delta_{-}(\lambda) \Delta_{+}(\lambda)
$$

where $\Delta_{-}$and $\Delta_{+}$are the characteristic functions of certain retarded and advanced equations, namely

$$
\Delta_{-}(\lambda)=\lambda-\int_{-1}^{0} e^{\lambda \theta} d \eta_{-}(\theta) \text { and } \Delta_{+}(\lambda)=\lambda-\int_{0}^{1} e^{\lambda \theta} d \eta_{+}(\theta)
$$

and where $\Psi$ is a polynomial of degree 1 which is needed as a correction factor to balance the growth rates on both sides of equation (6.3). By means of this result, we can obtain a solution of (6.1) by solving the retarded functional differential equation (RFDE):

$$
\dot{x}(t)=\int_{-1}^{0} x(t+\theta) d \eta_{-}(\theta) .
$$

The theory of RFDE gives the following result: 
Proposition 6.1. If $\Delta_{-}(\lambda)$ has no zeros in the right half plane $\{\lambda \mid \operatorname{Re}(\lambda) \geq 0\}$, all solutions of the RFDE (6.4) converge to zero exponentially as $t \rightarrow+\infty$.

For more information on RFDE, we refer the reader to Bellman and Cooke [1], Hale [7] and Hale and Verduyn Lunel [8].

Acknowledgements. We are very grateful for comments from Semyon Malamud (ETH, Zürich). We also thank the European Social Funds (ESF) and the Advanced Mathematical Methods for Finance (AMaMeF) programme of the European Science Foundation (ESF) for financial support during this work.

\section{References}

[1] R. Bellman and K. L. Cooke, Differential-Difference Equations, Academic Press, New York, 1963.

[2] J. M. Bismut, Conjugate convex functions in optimal stochastic control, J. Math. Anal. Appl. 44 (1973), 384-404.

[3] J. M. Bismut, Growth and optimal intertemporal allocations of risks, J. Economic Theory, 10 (1975), 239-287.

[4] R. Boucekkine, M. Germain, and O. Licandro, Replacement echoes in the vintage capital growth model, J. Economic Theory 74 (1997), 333-348.

[5] J. C. Cox and C. F. Huang, Optimal consumption and portfolio policies when asset prices follow a diffusion process, J. Economic Theory 49 (1989), 33-83.

[6] H. d'Albis and E. Augeraud-Véron, Balanced cycles in an OLG model with a continuum of finitely-lived individuals, Economic Theory, 2005.

[7] J. K. Hale, Theory of Functional Differential Equations, Springer-Verlag, New York, 1977.

[8] J. K. Hale and S. M. Verduyn Lunel, Introduction to Functional Differential Equations. Springer-Verlag, New York, 1993.

[9] I. Karatzas, P. Lakner, J. Lehoczky, and S. Shreve, Dynamic equilibrium in a multi-agent economy: construction and uniqueness, in: Stochastic Analysis: Liber Amicorum for Moshe Zakai (E. Meyer-Wolf, A. Schwartz and O. Zeitouni, eds.), 1991, 245-272.

[10] I. Karatzas, J. Lehoczky, and S. Shreve, Optimal portfolio and consumption decisions for a "small investor" in a finite horizon, SIAM J. Control \& Optimization 25 (1987), $1557-1586$.

[11] I. Karatzas, J. Lehoczky, and S. Shreve, Existence and uniqueness of multi-agent equilibrium in a stochastic, dynamic consumption/investment model, Math. Operations Research 15 (1990), 80-128.

[12] I. Karatzas, J. Lehoczky, and S. Shreve, Equilibrium models with singular asset prices, Mathematical Finance 1 (1991), 11-29.

[13] I. Karatzas and S. E. Shreve, Methods of Mathematical Finance. Springer, 1998.

[14] O. Kim, Balanced equilibrium in a consumption loans model, J. Economic Theory 29 (1983), 339-346.

[15] J. Lehoczky, S. Sethi, and S. Shreve, Optimal consumption and investment policies allowing consumption constraints and bankruptcy, Math. Operations Research 8 (1983), 613-636. 
[16] J. Mallet-Paret and S. V. Verduyn-Lunel, Exponential dichotomies and Wiener-Hopf factorizations for mixed-type functional differential equations, J. Differential Equations, to appear.

[17] R. C. Merton, Lifetime portfolio selection under uncertainty: The continuous-time case. Review of Economics and Statistics 51 (1969), 247-257.

[18] R. C. Merton, Optimum consumption and portfolio rules in a continuous-timt model, J. Economic Theory 3 (1971), 373-413.

[19] H. M. Polemarchakis and S. Demichelis, Life-span and the determinacy of equilibrium in economies of overlapping generations, Univ. Catholique de Louvain, Center for Operations Research and Economics, 2000. 